\title{
Interactive comment on "Performance evaluation of multiple satellite rainfall products for Dhidhessa River Basin (DRB), Ethiopia” by Gizachew Kabite Wedajo et al.
}

\section{Gizachew Kabite Wedajo et al.}

kabiteg@gmail.com

Received and published: 21 December 2020

Response to Reference 1 (RC1) Major comments (1) The novelty of the work In contrast to several previous studies on SREs evaluation, the present study combined statistical and hydrological performance evaluation in data scarce river basin of upper Blue Nile basin, the Dhidhessa River Basin. This method is important to identify SREs that better detect and estimate rainfall, and select application specific rainfall products such as for hydrologic and climate change studies. The results of this study also highlights seasonal dependence of rainfall detection and hydrologic performance capability of SREs for DRB and similar basins in Ethiopia. In addition, the performance of IMERG6, 
which is the latest SREs product, was evaluated for Ethiopian basin for the first time and the results showed that the product better performed for the DRB in estimating rainfall and predicting streamflow. Therefore, the new insights of this study include: i) the SREs evaluation was done by combining statistical and hydrological modelling methods; ii) the SREs considered in this study are the latest products reported best in different studies, and IMERG6 is the most recent product evaluated in Ethiopian basin's for the first time in this study and iii) the rainfall detection and estimation as well as streamflow prediction capability of SREs is dependent on seasons. The study results of this study are of interest to both research communities and decision-makers, and this paper has made a good contribution to improve understanding of the latest SREs for Ethiopia and the DRB. Therefore, based on your comments, I have modified the discussion and conclusion parts with the above points. (2) Categorical metrics Categorical metrics were defined based on the zero or non-zero rainfall for the individual months to evaluate rainfall detection capability of the SREs but not for the monthly average rainfall. Therefore, rainfall detection capability of the SRES were evaluated based on Probability of Detection (POD), False Aram Ratio (FAR) and Critical success Index (CSI). (2) Hydrological simulation I have incorporated the following points in the revised manuscript. The groundwater parameters can have a strong effect on the amount of streamflow that can cause over or underestimation of streamflow. For this reason, the validation of streamflow was sorely dependent on the rainfall products. Minor comments * found that there are some typos, grammatical errors, and inconsistent tense throughout the manuscript, please pore over them. Line 118: Change "distributed" to "semi-distributed". Corrected accordingly. Line 460: Change "stremflows" to "streamflow". Corrected. Figure 1: The legends are too small to read them. Please remove the road lines (and town symbols if appropriate). I recommend showing the subbasin boundary only for the upstream of the streamflow gauge station. Corrected as suggested. Table 1: Please check the data periods. I suggest using actual data periods used in the study instead of the entire periods. Corrected for the revised version. Table 2: The present form of the coefficient of determination ( $r 2$ ) is the Pearson correlation

Interactive comment
Printer-friendly version

Discussion paper

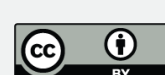


(r). Lines 258-263: Again, please specify the category. Corrected accordingly in the revised manuscript. Figure 2: Can you use the same color limits for all products? I also AMTD recommend showing the standard deviation maps. The SREs products have different minimum and maximum limit values. Therefore, it is not possible to make the same color limits for all products. The aim of this map is to show the capability of the products in capturing spatial rainfall variability and how the minimum and maximum values limits varies among the products. Lines 390-391: Please describe the details of these parameters. The meaning of the parameters are included in the revised version of the manuscript. Table 4: What does the fit value mean here (e.g., negative curve number)? The best fit values were multiplied by (1+ given value) for the parameters with r-prefix and replaced by the given value for the parameters with v-prefix. Lines 399-400: Does the observed rainfall have that uncertainty too? The overestimation of high streamflow could be also due to SWAT model errors in addition to the rainfall estimation uncertainty of SREs and measurement errors of the observed rainfall.

Interactive comment on Atmos. Meas. Tech. Discuss., doi:10.5194/amt-2020-355, 2020.

Interactive comment

Discussion pap 


\section{AMTD}

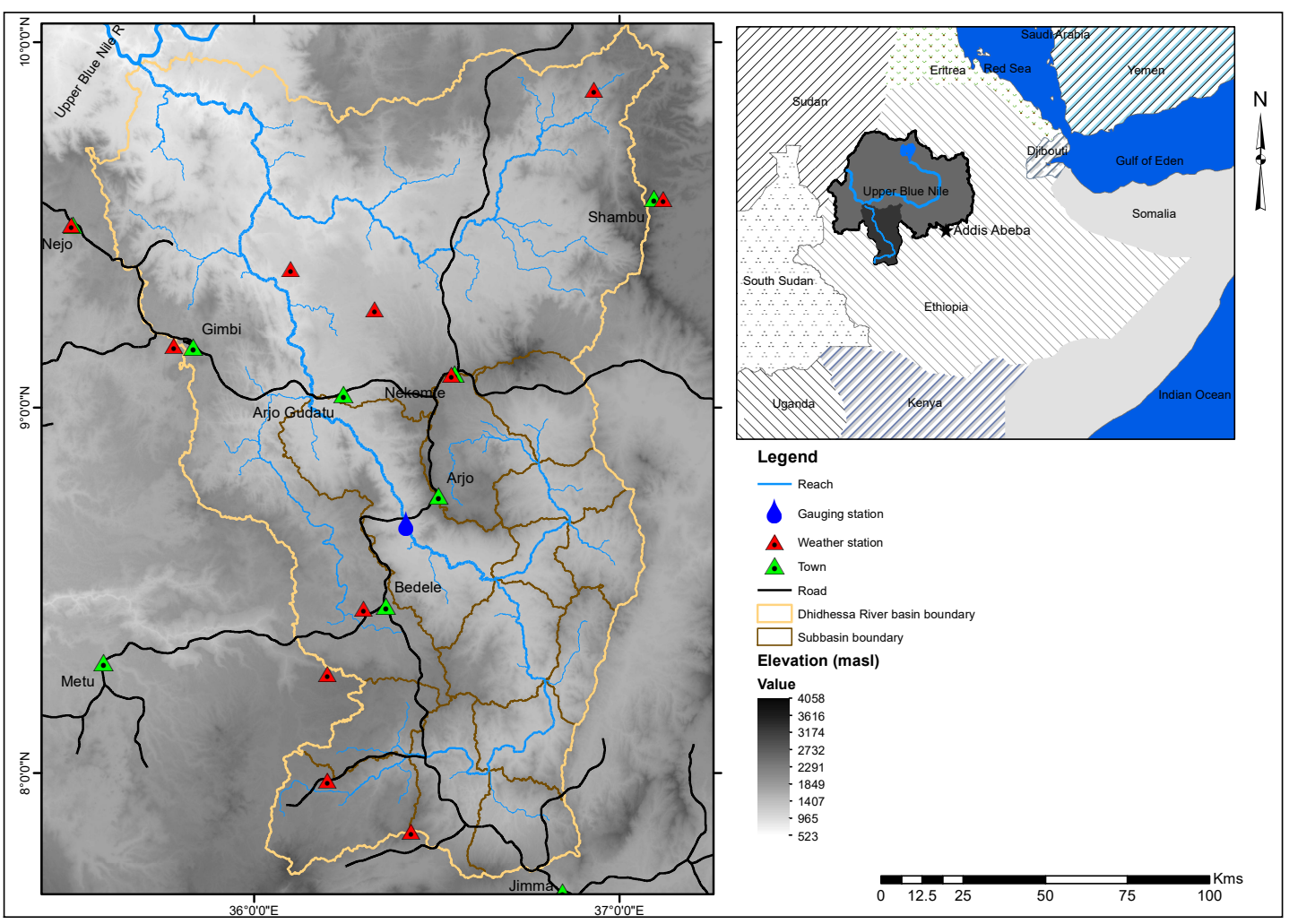

Interactive comment

Fig. 1. Location Map

Discussion paper 